\title{
Nachweis von Autoantikörpern bei Zöliakie mittels iRIfS
}

\author{
Barbara Schwarz, Günther Proll, Günter Gauglitz \\ Eberhard Karls Universität Tübingen, Auf der Morgenstelle 18, 72076 Tübingen \\ Barbara.schwarz@uni-tuebingen.de
}

\section{Zusammenfassung}

In der hier vorgestellten Arbeit wird die Detektion von Peptid-Antikörperwechselwirkungen mittels bildgebender Reflektometrischer Interferenzspektroskopie (iRIfS), einer parallelisierten direkt-optischen Methode untersucht. Diese Methode ist eine Weiterentwicklung der Reflektometrischen Interferenzspektroskopie (RIfS) [1] und ermöglicht parallele biomolekulare Interaktionsanalysen im Arrayformat mit Arrays bis zu 1000 Spots pro 1,5 x 1,5 cm². Die Untersuchungen werden am Beispiel der Autoimmunkrankheit Zöliakie durchgeführt. Neben der parallelisierten Detektion der Antikörper-Peptid Wechselwirkung wird auf die Entwicklung einer geeigneten Oberflächenchemie eingegangen, sowie erste Messungen mit realen Patientenproben gezeigt.

\section{Einleitung und Hintergrund}

Bei der Diagnose von Autoimmunkrankheiten spielt die Detektion von Autoantikörpern eine wichtige Rolle. Der Nachweis und die Charakterisierung von Autoantikörpern ermöglicht eine verbesserte Diagnose, Aussagen über den Krankheitsverlauf und gegebenenfalls eine antigenspezifische, individualisierte Therapie. Oftmals erfolgen der Nachweis und das Epitopmapping der Autoantikörper mittels Peptidarrays [2]. Verwendet man diese in Kombination mit zeitaufgelösten Nachweismethoden, können kinetische und thermodynamische Konstanten bestimmt und somit biomolekulare Wechselwirkungen charakterisiert werden. Ein weiterer Vorteil zeitaufgelöster Methoden, im Gegensatz zu Endpunktmethoden, liegt darin auch schwach-affine Antikörper-Antigen Interaktionen nachweisen zu können. Beide genannten Punkte sind vorteilhaft für die Diagnose und die Überwachung des Krankheitsverlaufs von Autoimmunkrankheiten.

Zöliakie ist eine Erkrankung des Darmtraktes, die durch das in Weizen und verwandten Getreidearten enthaltene Gluten hervorgerufen wird. Dabei kommt es zu Entzündungsreaktionen der Darmschleimhaut. In dem hervorgerufenen Entzündungsprozess werden neben anti-Gliadin Antikörpern auch Autoantikörper gegen das in der Darmschleimhaut vorkommende Enzym Transglutaminase gebildet [3]. Das System Transglutaminase - Anti-Transglutamianse Antikörper wurde in der vorliegenden Arbeit als Modellsystem für eine Autoimmunkrankheit verwendet und Wechselwirkungen zwischen verschiedenen TransglutaminasePeptiden und einem Anti-Transglutaminase-Antikörper untersucht.

\section{Reflektometrische Interferenzspektroskopie (RIfS) und bildgebenden Reflektometrischen Interferenzspektroskopie (iRIfS)}

Zur Detektion der Peptid-Antikörper Wechselwirkungen wurde die direkt-optische markierungsfreie Methode der Reflektometrischen Interferenzspektroskopie verwendet. Sie erlaubt zeitaufgelöste Messungen und somit die Bestimmung von kinetischen und thermodynamischen Konstanten.

RIfS beruht auf Reflektion und Interferenz von Weißlicht an dünnen Schichten. Weißlicht wird senkrecht in ein Schichtsystem eingestrahlt, dabei kommt es an jeder Phasengrenze zu Reflektion und Transmission von Teilstrahlen. Die reflektierten Teilstrahlen interferieren miteinander und überlagern sich zu einem, für das jeweilige Schichtsystem charakteristischen Interferenzspektrum. Bei einer Änderung des Schichtsystems z.B. durch Anlagerung von Analytmolekülen an den Sensor, kommt es zu einer Verschiebung des Spektrums in 
Abhängigkeit der sogenannten optischen Schichtdicke. Die optische Schichtdicke ist das Produkt aus physikalischer Schichtdicke und Brechungsindex. Da die Temperaturabhängigkeit beider Parameter zueinander invers proportional ist, ist RIfS im Gegensatz zu Evaneszenzfeld-Methoden wie Oberflächenplasmonresonanz, weitgehend temperaturunabhängig. Aus der Verschiebung des Spektrums können dann zeitaufgelöste Bindungskurven gewonnen werden.

Neben der spektralen Auswertung können Bindungskurven auch über die Änderung der Intensität bei einer Wellenlänge berechnet werden. Dies ermöglicht entweder einen einfacheren Messaufbau, da das Weißlicht durch eine LED und das Spektrometer durch eine Photodiode ersetzt werden kann oder eine Parallelisierung der Messungen. Hierbei wird ebenfalls eine LED als Lichtquelle verwendet, die Detektion jedoch erfolgt über den CCD-Chip einer Kamera. Dieser Messaufbau erlaubt unter anderem die Messung von Protein - und Peptidarrays und eignet sich somit für die Untersuchung von verschiedenen Peptiden mit einem antiTransglutaminase Antikörper.

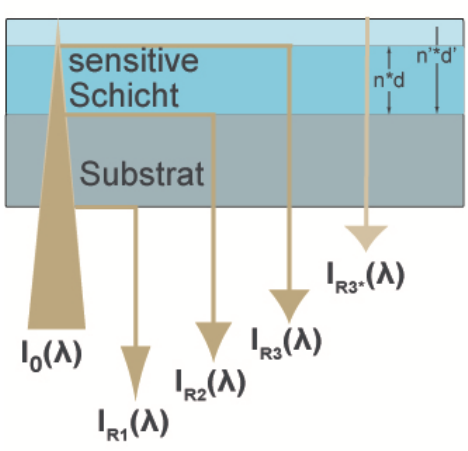

Reflektion an dünnen Schichten

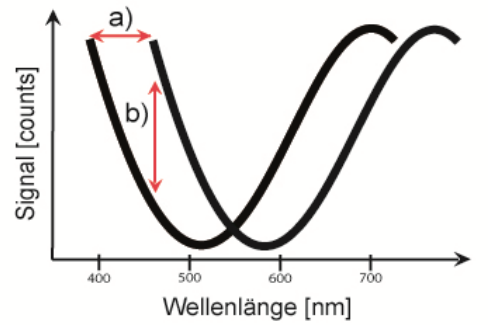

Interferenzspektrum und Auswertemodi

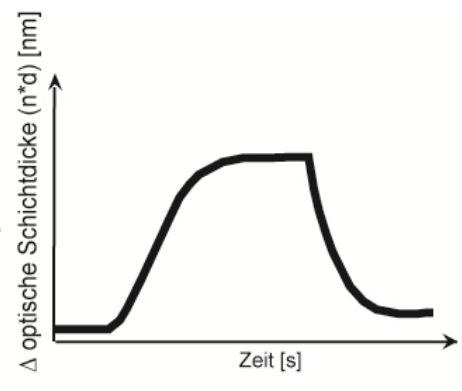

Zeitaufgelöste Bindungskurve

Bild 1 Prinzip der Reflektometrischen Interferenz Spektroskopie:

Licht wird senkrecht in ein Schichtsystem eingestrahlt und die Teilstrahlen mehrfach transmittiert und reflektiert. Die reflektierten Teilstrahlen interferieren zu einem charakteristischen Interferenzspektrum, die Berechnung der Bindungskurve kann entweder aus der Verschiebung des Minimums (a) oder durch die Änderung der Intensität bei einer Wellenlänge (b) berechnet werden

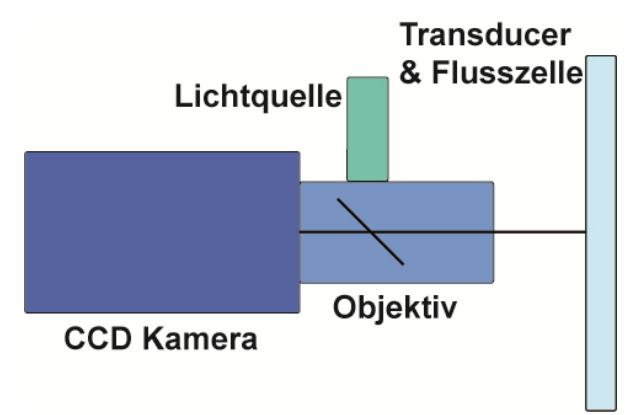

Bild 2 Messaufbau der bildgebenden Reflektometrischen Interferenzspektroskopie: Als Lichtquelle dient eine LED, als Detektor eine CCD-Kamera

\section{Ergebnisse}

\section{Oberflächenchemie}

Da markierungsfreie Methoden jedes Bindungsereignis an der Sensoroberfläche detektieren, muss ein besonderes Augenmerk in der Assayentwicklung auf die verwendeten Oberflächenchemie gelegt werden. Dabei ist es wichtig, die unspezifischen Bindungen zu minimieren ohne die spezifischen Bindungen zu beeinflussen. Dazu wurden zwei Oberflächenpolymere ausgewählt und verglichen. Zum einen wurde ein Aminodextran (AMD) basiertes Hydrogel verwendet, das sich in der Regel durch eine hohe 
Oberflächenbeladung auszeichnet, zum anderen eine Beschichtung aus Polyethylenglycol, das meist eine geringere Oberflächenbeladung, kombiniert mit geringeren unspezifischen Wechselwirkungen, ermöglicht. Diese beiden Polymere wurden hinsichtlich ihrer Eignung zur Beschichtung der Sensoroberfläche für die Peptidarrays getestet.

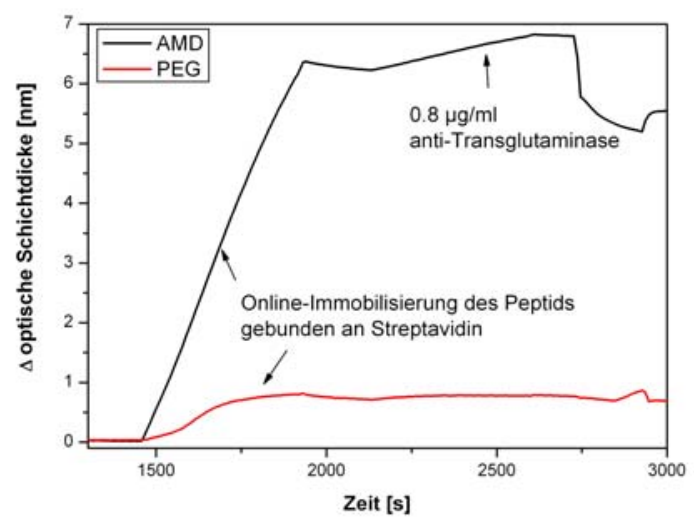

Bild 3 Vergleich der verschiedenen Oberflächenpolymere hinsichtlich ihrer Eignung für die Präparation der Sensoroberfläche

Da letztendlich Autoantikörper in Serumproben nachgewiesen werden sollen, müssen die Peptide, die verschiedene Epitope präsentieren, auf der Oberfläche immobilisiert werden. Am geeignetsten hierfür erschien die weitgehend gerichtete Online-Immobilisierung von Peptiden über einen Biotin-Linker an die Oberfläche. Folgende Punkte wurden hierbei untersucht a) Anbindung der mit Streptavidin vorinkubierten Peptide an die biotinylierte Polymeroberfläche und nachfolgende Anbindung des Antikörpers und b) die unspezifische Bindung von fötalem Kälberserum (FCS) an die Sensoroberfläche als Modell für spätere Serummessungen.

Tafel 1 Vergleich der unspezifischen Bindung mit FCS an AMD und PEG - Oberflächen

\begin{tabular}{l|l}
\hline AMD & PEG \\
\hline $1,73 \pm 0,42 \mathrm{~nm}$ & $1,78 \pm 0,15 \mathrm{~nm}$ \\
\hline
\end{tabular}

Die Messungen ergaben für Aminodextran im Gegensatz zu PEG ein höheres Bindungssignal, sowohl für die Online-Immobilisierung des Peptids, als auch die Antikörperanbindung. Die unspezifische Wechselwirkung der Oberfläche mit FCS schwankte bei AMD Oberflächen stärker, als bei PEG, liegt aber bei beiden Polymeren in einem ähnlichen Bereich. Aufgrund dieser Ergebnisse wurde AMD als Beschichtungspolymer für weitere Versuche ausgewählt.

\section{iRIfS Messungen}

Für die parallelisierten Messungen wurde ein Array aus 5 konsekutiven Peptiden auf AMD gespottet, die ein Epitop des anti-Transglutaminase Antikörpers umfassen. Die Wechselwirkungen des Antikörpers mit den verschiedenen Peptiden wurden mittels iRIfS Messungen detektiert. Die gemessenen Signalhöhen korrelieren mit den erwarteten Affinitäten [4]. 


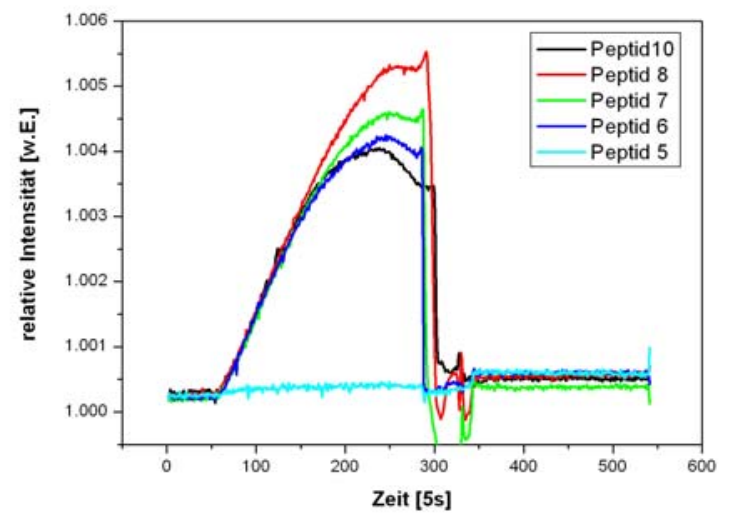

Peptid 5: YPEGSSEEREAFTRAN

Peptid 6: PEGSSEEREAFTRANH

Peptid 7: $\quad$ EGSSEEREAFTRANHL

Peptid 8: $\quad$ GSSEEREAFTRANHLN

Peptid 10: $\quad$ SEEREAFTRANHLNKL

Bild 3 a) gespottete Peptidsequenzen b) iRIfS- Messung: Anbindung des anti-Transglutaminaseantikörpers an die Peptide. Die Bindungssignale korrelieren mit den zu erwartenden Affinitäten.

\section{Serum Messungen}

Darüber hinaus wurden Messungen mit realen Patientenseren durchgeführt. Dabei konnte mittels RIfS gezeigt werden, dass mit den entsprechenden Peptidoberflächen zwischen Negativ- und Positivseren unterschieden werden kann.

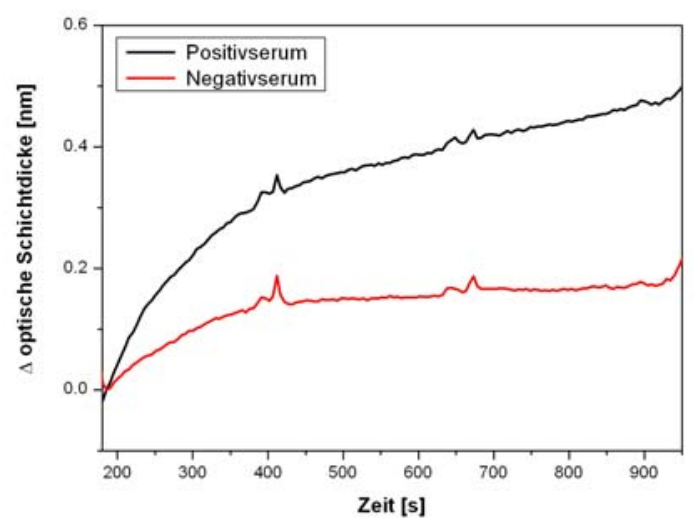

Bild 4 Messung mit realen Patientenseren. Positives Serum zeigt ein deutliches Bindungssignal gegenüber Negativserum

\section{Ausblick}

In den folgenden Messungen sollen parallelisiert Affinitätskonstanten mittels des iRIfS-Systems bestimmt und Messungen mit realen Seren am iRIfS-System durchgeführt werden. Zudem soll der Array um weitere relevante Epitope erweitert werden.

\section{Literatur}

[1] Gauglitz, G.; Proll, G., Adv Biochem Engin/Biotechnol, 2008, 109, 395-432

[2] Sharp,V., Utz, P.J., Nature Clinical Practice 2007, 3(2), 96-103.

[3] Dietrich, W., Ehnis,T., Bauer, M., Donner, P., Volta, U., Riecken, E., Schuppan, D., Nature Medicine, 1997, 3(7), 797-801

[4] Kröger, K. , Bauer, J, v.d. Mülbe, F., Fleckenstein, B., Rademann, R., Jung, G., Gauglitz, G., Epitope-mapping of transglutaminase with labelfree detection Biosensors\&Bioelectronics 17 (2002), 937944. 